\title{
Continuity for multifunctions in ideal topological spaces
}

\author{
CHAWALIT BOONPOK \\ Mathematics and Applied Mathematics Research Unit \\ Department of Mathematics, Faculty of Science, Mahasarakham University \\ Maha Sarakham, 44150 \\ THAILAND \\ PRAPART PUE-ON \\ Mathematics and Applied Mathematics Research Unit \\ Department of Mathematics, Faculty of Science, Mahasarakham University \\ Maha Sarakham, 44150 \\ THAILAND
}

\begin{abstract}
This article presents the concept of $\imath^{\star}$-continuous multifunctions in ideal topological spaces. Especially, several characterizations of $\imath^{\star}$-continuous multifunctions are investigated. Moreover, the relationships between $\imath^{\star}$-continuity and the other types of continuity for multifunctions are established.
\end{abstract}

Key-Words: $\star$-open set, $\imath^{\star}$-continuous multifunction, almost $\imath^{\star}$-continuous multifunction, weakly $\imath^{\star}$-continuous multifunction

Received: April 8, 2020. Revised: December 5, 2020. Accepted: December 19, 2020. Published: December 29, 2020.

\section{Introduction}

The branch of mathematics called topology is concerned with all questions directly or indirectly related to continuity. Continuity is an important concept for the study and investigation in the theory of classical point set topology. Generalization of this concept by using weaker forms of open sets. The notion of weak continuity due to Levine [13] is one of the most important weak forms of continuity in topological spaces. Rose [17] introduced the notion of subweakly continuous functions and investigated the relationships between subweak continuity and weak continuity. The present authors introduced and studied weakly quasi-continuous multifunctions [14], weakly precontinuous multifunctions [15] and weakly $\beta$-continuous multifunctions [16]. These multifunctions have similar properties. The analogy in their definitions and results suggests the need of formulating a unified theory.

The concept of ideals in topological spaces has been introduced and studied by Kuratowski [12] and Vaidyanathswamy [19]. The topology $\tau$ of a space is enlarged to a topology $\tau^{\star}$ using an ideal $\mathscr{I}$ whose members are disjoint with the members of $\tau$. Every topological space is an ideal topological space and all the results of ideal topological spaces are generalizations of the results established in topological spaces.
Some early applications of ideal topological spaces can be found in various branches of mathematics, like a generalization of Cantor-Bendixson theorem by Freud [7], or in measure theory by Scheinberg [18]. In 1990, Janković and Hamlett [11] introduced the notion of $\mathscr{I}$-open sets in ideal topologial spaces. Abd El-Monsef et al. [1] further investigated $\mathscr{I}$-open sets and $\mathscr{I}$-continuous functions. Later, several authors studied ideal topological spaces giving several convenient definitions. Some authors obtained decompositions of continuity. For instance, Açikgöz et al. [2] introduced and investigated the notions of weakly$\mathscr{I}$-continuous and weak ${ }^{\star}-\mathscr{I}$-continuous functions in ideal topological spaces. Donthev [6] introduced the notion of pre- $\mathscr{I}$-open sets and obtained a decomposition of $\mathscr{I}$-continuity. Hatir and Noiri [10] introduced the notions of semi- $\mathscr{I}$-open sets, $\alpha-\mathscr{I}$-open sets and $\beta-\mathscr{I}$-open sets via idealization and using these sets obtained new decompositions of continuity. In 2005, Hatir and Noiri [9] investigated further properties of semi- $\mathscr{I}$-open sets and semi- $\mathscr{I}$-continuity. Hatir et al. [8] introduced and investigated the notions of strong $\beta-\mathscr{I}$-open sets and strongly $\beta$ - $\mathscr{I}$-continuous functions.

The purpose of the present article is to introduce the notions of $\imath^{\star}$-continuous multifunctions, almost $\imath^{\star}$-continuous multifunctions and weakly $\imath^{\star}$ continuous multifunctions. In particular, some char- 
acterizations of $\imath^{\star}$-continuous multifunctions, almost $\imath^{\star}$-continuous multifunctions and weakly $\imath^{\star}$ continuous multifunctions are investigated. Furthermore, the relationships between $\imath^{\star}$-continuity, almost $\imath^{\star}$-continuity and weak $\imath^{\star}$-continuity are discussed.

\section{Preliminaries}

Throughout the present paper, spaces $(X, \tau)$ and $(Y, \sigma)$ (or simply $X$ and $Y$ ) always mean topological spaces on which no separation axioms are assumed unless explicitly stated. In a topological space $(X, \tau)$, the closure and the interior of any subset $A$ of $X$ will denoted by $\mathrm{Cl}(A)$ and $\operatorname{Int}(A)$, respectively. An ideal $\mathscr{I}$ on a topological space $(X, \tau)$ is a nonempty collection of subsets of $X$ satisfying the following properties: (1) $A \in \mathscr{I}$ and $B \subseteq A$ implies $B \in \mathscr{I}$; (2) $A \in \mathscr{I}$ and $B \in \mathscr{I}$ implies $A \cup B \in \mathscr{I}$. A topological space $(X, \tau)$ with an ideal $\mathscr{I}$ on $X$ is called an ideal topological space and is denoted by $(X, \tau, \mathscr{I})$. For an ideal topological space $(X, \tau, \mathscr{I})$ and a subset $A$ of $X, A^{\star}(\mathscr{I})$ is defined as follows: $A^{\star}(\mathscr{I})=\{x \in X: U \cap A \notin \mathscr{I}$ for every open neighbourhood $U$ of $x\}$. In case there is no chance for confusion, $A^{\star}(\mathscr{I})$ is simply written as $A^{\star}$. In [12], $A^{\star}$ is called the local function of $A$ with respect to $\mathscr{I}$ and $\tau$ and $\mathrm{Cl}^{\star}(A)=A^{\star} \cup A$ defines a Kuratowski closure operator for a topology $\tau^{\star}(\mathscr{I})$. For every ideal topological space $(X, \tau, \mathscr{I})$, there exists a topology $\tau^{\star}(\mathscr{I})$ finer than $\tau$, generated by $\mathscr{B}(\tau, \mathscr{I})=\left\{U-I_{0} \mid U \in \tau\right.$ and $\left.I_{0} \in \mathscr{I}\right\}$, but in general $\mathscr{B}(\tau, \mathscr{I})$ is not always a topology [11]. A subset $A$ is said to be $\star$-closed [11] if $A^{\star} \subseteq A$. The complement of a $\star$-closed set is said to be $\star$-open. The interior of a subset $A$ in $\left(X, \tau^{\star}(\mathscr{I})\right)$ is denoted by $\operatorname{Int}^{\star}(A)$.

By a multifunction $F: X \rightarrow Y$, we mean a pointto-set correspondence from $X$ into $Y$, and we always assume that $F(x) \neq \emptyset$ for all $x \in X$. For a multifunction $F: X \rightarrow Y$, following [3], we shall denote the upper and lower inverse of a set $B$ of $Y$ by $F^{+}(B)$ and $F^{-}(B)$, respectively, that is,

$$
F^{+}(B)=\{x \in X \mid F(x) \subseteq B\}
$$

and $F^{-}(B)=\{x \in X \mid F(x) \cap B \neq \emptyset\}$. In particular, $F^{-}(y)=\{x \in X \mid y \in F(x)\}$ for each point $y \in Y$. For each $A \subseteq X, F(A)=\cup_{x \in A} F(x)$. Then $F$ is said to be surjection if $F(X)=Y$, or equivalent, if for each $y \in Y$ there exists $x \in X$ such that $y \in F(x)$ and $F$ is called injection if $x \neq y$ implies $F(x) \cap F(y)=\emptyset$.

Let $\mathscr{P}(X)$ be the collection of all nonempty subsets of $X$. For any $\star$-open set $V$ of an ideal topological space $(X, \tau, \mathscr{I})$, we denote

$$
V^{+}=\{B \in \mathscr{P}(X) \mid B \subseteq V\}
$$

and $V^{-}=\{B \in \mathscr{P}(X) \mid B \cap V \neq \emptyset\}$.

Definition 1. [5] A subset $A$ of an ideal topological space $(X, \tau, \mathscr{I})$ is said to be:

(i) $R-\mathscr{I}^{\star}$-open if $A=\operatorname{Int}^{\star}\left(C l^{\star}(A)\right)$;

(ii) $R-\mathscr{I}^{\star}$-closed if its complement is $R-\mathscr{I}^{\star}$-open;

(iii) $\mathscr{I}^{\star}$-preopen if $A \subseteq \operatorname{Int}^{\star}\left(C l^{\star}(A)\right)$;

(iv) $\mathscr{I}^{\star}$-preclosed if its complement is $\mathscr{I}^{\star}$-preopen.

Definition 2. [4] A subset $A$ of an ideal topological space $(X, \tau, \mathscr{I})$ is said to be:

(i) semi- $\mathscr{I}^{\star}$-open if $A \subseteq C l^{\star}\left(\operatorname{Int^{\star }}(A)\right)$;

(ii) semi- $\mathscr{I}^{\star}$-closed if its complement is semi- $\mathscr{I}^{\star}$ open;

(iii) semi- $\mathscr{I}^{\star}$-preopen if $A \subseteq C l^{\star}\left(\operatorname{Int^{\star }}\left(C l^{\star}(A)\right)\right)$;

(iv) semi- $\mathscr{I}^{\star}$-preclosed if its complement is semi$\mathscr{I}^{\star}$ - preopen.

\section{On characterizations of $\imath^{\star}$ - continuous multifunctions}

In this section, we introduce the concept of $\imath^{\star}$ continuous multifunctions and investigate some characterizations of $\imath^{\star}$-continuous multifunctions.

Definition 3. A multifunction $F:(X, \tau, \mathscr{I}) \rightarrow$ $(Y, \sigma, \mathscr{J})$ is said to be $\imath^{\star}$-continuous if for each $x \in X$ and each $\star$-open sets $V_{1}, V_{2}$ of $Y$ such that $F(x) \in V_{1}^{+} \cap V_{2}^{-}$, there exists $a \star$-open set $U$ of $X$ containing $x$ such that $F(U) \subseteq V_{1}$ and $F(z) \cap V_{2} \neq \emptyset$ for every $z \in U$.

Theorem 4. For a multifunction $F:(X, \tau, \mathscr{I}) \rightarrow$ $(Y, \sigma, \mathscr{J})$, the following properties are equivalent:

(1) F is $\imath^{\star}$-continuous;

(2) $x \in \operatorname{Int}^{\star}\left[F^{+}\left(V_{1}\right) \cap F^{-}\left(V_{2}\right)\right]$ for every $\star$-open set $V_{1}, V_{2}$ of $Y$ such that $F(x) \subseteq V_{1}$ and

$$
F(x) \cap V_{2} \neq \emptyset ;
$$

(3) $F^{+}\left(V_{1}\right) \cap F^{-}\left(V_{2}\right)$ is $\star$-open in $X$ for any $\star$-open set $V_{1}, V_{2}$ of $Y$;

(4) $F^{-}\left(K_{1}\right) \cup F^{+}\left(K_{2}\right)$ is $\star$-closed in $X$ for any $\star-$ closed sets $K_{1}, K_{2}$ of $Y$; 
(5)

$$
\begin{aligned}
& C l^{\star}\left[F^{-}\left(B_{1}\right) \cup F^{+}\left(B_{2}\right)\right] \\
& \subseteq F^{-}\left(C l^{\star}\left(B_{1}\right)\right) \cup F^{+}\left(C l^{\star}\left(B_{2}\right)\right)
\end{aligned}
$$

for any subsets $B_{1}, B_{2}$ of $Y$;

(6)

$$
\begin{aligned}
& F^{-}\left(\operatorname{Int}^{\star}\left(B_{1}\right)\right) \cap F^{+}\left(\operatorname{Int}^{\star}\left(B_{2}\right)\right) \\
& \subseteq \operatorname{Int}^{\star}\left[F^{-}\left(B_{1}\right) \cap F^{+}\left(B_{2}\right)\right]
\end{aligned}
$$

for any subsets $B_{1}, B_{2}$ of $Y$.

Proof. (1) $\Rightarrow(2)$ : Let $V_{1}, V_{2}$ be any $\star$-open sets of $Y$ such that $F(x) \subseteq V_{1}$ and $F(x) \cap V_{2} \neq \emptyset$. Then, there exists a $\star$-open set $U$ of $X$ containing $x$ such that $F(U) \subseteq V_{1}$ and $F(z) \cap V_{2} \neq \emptyset$ for every $z \in U$. Thus, $U \subseteq F^{+}\left(V_{1}\right) \cap F^{-}\left(V_{2}\right)$ and hence

$$
x \in \operatorname{Int}^{\star}\left[F^{+}\left(V_{1}\right) \cap F^{-}\left(V_{2}\right)\right] .
$$

$(2) \Rightarrow(3)$ : Let $V_{1}, V_{2}$ be any $\star$-open sets of $Y$ and $x \in F^{+}\left(V_{1}\right) \cap F^{-}\left(V_{2}\right)$. Then $F(x) \subseteq V_{1}$ and $F(x) \cap V_{2} \neq \emptyset$. By (2), we have

$$
x \in \operatorname{Int}^{\star}\left[F^{+}\left(V_{1}\right) \cap F^{-}\left(V_{2}\right)\right]
$$

and hence

$$
F^{+}\left(V_{1}\right) \cap F^{-}\left(V_{2}\right) \subseteq \operatorname{Int}^{\star}\left[F^{+}\left(V_{1}\right) \cap F^{-}\left(V_{2}\right)\right] .
$$

This shows that $F^{+}\left(V_{1}\right) \cap F^{-}\left(V_{2}\right)$ is $\star$-open in $X$.

$(3) \Rightarrow(4)$ : This follows from the fact that $F^{-}(Y-B)=X-F^{+}(B)$ and

$$
F^{+}(Y-B)=X-F^{-}(B)
$$

for every subset $B$ of $Y$.

$(4) \Rightarrow(5)$ : Let $B_{1}, B_{2}$ be any subsets of $Y$. Then $\mathrm{Cl}^{\star}\left(B_{1}\right)$ and $\mathrm{Cl}^{\star}\left(B_{2}\right)$ are $\star$-closed in $Y$. By (4), we have

$$
\begin{aligned}
& \mathrm{Cl}^{\star}\left[F^{-}\left(B_{1}\right) \cup F^{+}\left(B_{2}\right)\right] \\
& \subseteq \mathrm{Cl}^{\star}\left[F^{-}\left(\mathrm{Cl}^{\star}\left(B_{1}\right)\right) \cup F^{+}\left(\mathrm{Cl}^{\star}\left(B_{2}\right)\right)\right] \\
& =F^{-}\left(\mathrm{Cl}^{\star}\left(B_{1}\right)\right) \cup F^{+}\left(\mathrm{Cl}^{\star}\left(B_{2}\right)\right) .
\end{aligned}
$$

$(5) \Rightarrow(6)$ : Let $B_{1}, B_{2}$ be any subsets of $Y$. By (5), we have

$$
\begin{aligned}
& X-\operatorname{Int}^{\star}\left[F^{-}\left(B_{1}\right) \cap F^{+}\left(B_{2}\right)\right] \\
& =\mathrm{Cl}^{\star}\left[X-\left[F^{-}\left(B_{1}\right) \cap F^{+}\left(B_{2}\right)\right]\right] \\
& =\mathrm{Cl}^{\star}\left[\left(X-F^{-}\left(B_{1}\right)\right) \cup\left(X-F^{+}\left(B_{2}\right)\right)\right] \\
& =\mathrm{Cl}^{\star}\left[F^{+}\left(Y-B_{1}\right) \cup F^{-}\left(Y-B_{2}\right)\right] \\
& \subseteq F^{+}\left(\mathrm{Cl}^{\star}\left(Y-B_{1}\right)\right) \cup F^{-}\left(\mathrm{Cl}^{\star}\left(Y-B_{2}\right)\right) \\
& =F^{+}\left(Y-\operatorname{Int}^{\star}\left(B_{1}\right)\right) \cup F^{-}\left(Y-\operatorname{Int}^{\star}\left(B_{2}\right)\right) \\
& =\left[X-F^{-}\left(\operatorname{Int}^{\star}\left(B_{1}\right)\right)\right] \cup\left[X-F^{+}\left(\operatorname{Int}^{\star}\left(B_{2}\right)\right)\right] \\
& =X-\left[F^{-}\left(\operatorname{Int}^{\star}\left(B_{1}\right)\right) \cap F^{+}\left(\operatorname{Int}^{\star}\left(B_{2}\right)\right)\right]
\end{aligned}
$$

and hence

$$
\begin{aligned}
& F^{-}\left(\operatorname{Int}^{\star}\left(B_{1}\right)\right) \cap F^{+}\left(\operatorname{Int}^{\star}\left(B_{2}\right)\right) \\
& \subseteq \operatorname{Int}^{\star}\left[F^{-}\left(B_{1}\right) \cap F^{+}\left(B_{2}\right)\right] .
\end{aligned}
$$

$(6) \Rightarrow(1)$ : Let $x \in X$ and $V_{1}, V_{2}$ be any $\star$-open sets of $Y$ such that $F(x) \subseteq V_{1}$ and $F(x) \cap V_{2} \neq \emptyset$. By (6), we have

$$
F^{+}\left(V_{1}\right) \cap F^{-}\left(V_{2}\right) \subseteq \operatorname{Int}^{\star}\left[F^{+}\left(V_{1}\right) \cap F^{-}\left(V_{2}\right)\right] .
$$

Now, put $U=F^{+}\left(V_{1}\right) \cap F^{-}\left(V_{2}\right)$, then $U$ is a $\star$-open set of $X$ containing $x$ such that $F(U) \subseteq V_{1}$ and

$$
F(z) \cap V_{2} \neq \emptyset
$$

for each $z \in U$. This shows that $F$ is $\imath^{\star}$-continuous.

\section{On characterizations almost $\imath^{\star}$ - continuous multifunctions}

In this section, we introduce the concept of almost $\imath^{\star}$-continuous multifunctions and investigate several characterizations of such multifunctions.

Definition 5. A multifunction $F:(X, \tau, \mathscr{I}) \rightarrow$ $(Y, \sigma, \mathscr{J})$ is said to be almost $\imath^{\star}$-continuous if for each $x \in X$ and each $\star$-open set $V_{1}, V_{2}$ of $Y$ such that $F(x) \in V_{1}^{+} \cap V_{2}^{-}$, there exists $a \star$-open set $U$ of $X$ containing $x$ such that $F(U) \subseteq \operatorname{Int}\left(C l^{\star}\left(V_{1}\right)\right)$ and $F(z) \cap \operatorname{Int}\left(C l^{\star}\left(V_{2}\right)\right) \neq \emptyset$ for every $z \in U$.

Remark 6. For a multifunction $F:(X, \tau, \mathscr{I}) \rightarrow$ $(Y, \sigma, \mathscr{J})$, the following implication holds:

$$
\imath^{\star} \text {-continuity } \Rightarrow \text { almost } \imath^{\star} \text {-continuity. }
$$

The converse of the implication is not true in general. We give example for the implication as follows.

Example 7. Let $X=\{1,2,3\}$ with a topology $\tau=\{\emptyset,\{1\},\{2\},\{1,2\}, X\}$ and an ideal $\mathscr{I}=$ $\{\emptyset,\{3\}\}$. Let $Y=\{a, b, c\}$ with a topology $\sigma=$ $\{\emptyset,\{a\},\{a, b\}, Y\}$ and an ideal $\mathscr{J}=\{\emptyset,\{c\}\} . A$ multifunction $F:(X, \tau, \mathscr{I}) \rightarrow(Y, \sigma, \mathscr{J})$ is defined as follows: $F(2)=\{c\}$ and $F(1)=\{a\}$ and $F(3)=\{a, b\}$. Then $F$ is almost $\imath^{\star}$-continuous but $F$ is not $\imath^{\star}$-continuous.

Theorem 8. For a multifunction $F:(X, \tau, \mathscr{I}) \rightarrow$ $(Y, \sigma, \mathscr{J})$, the following properties are equivalent:

(1) $F$ is almost $\imath^{\star}$-continuous; 
(2)

$$
\begin{aligned}
& F^{+}\left(V_{1}\right) \cap F^{-}\left(V_{2}\right) \\
& \subseteq \operatorname{Int}^{\star}\left[F^{+}\left(\operatorname{Int}\left(C l^{\star}\left(V_{1}\right)\right)\right) \cap F^{-}\left(\operatorname{Int}\left(C l^{\star}\left(V_{2}\right)\right)\right)\right]
\end{aligned}
$$

for every $\star$-open sets $V_{1}, V_{2}$ of $Y$;

(3)

$$
\begin{aligned}
& C l^{\star}\left[F^{-}\left(C l\left(\operatorname{Int} t^{\star}\left(K_{1}\right)\right)\right) \cup F^{+}\left(C l\left(\operatorname{Int} t^{\star}\left(K_{2}\right)\right)\right)\right] \\
& \subseteq F^{-}\left(K_{1}\right) \cup F^{+}\left(K_{2}\right)
\end{aligned}
$$

for every $\star$-closed sets $K_{1}, K_{2}$ of $Y$;

(4)

$$
\begin{aligned}
& C l^{\star}\left[F^{-}\left(C l\left(\operatorname{Int}^{\star}\left(C l^{\star}\left(B_{1}\right)\right)\right)\right)\right. \\
& \left.\cup F^{+}\left(C l\left(\operatorname{Int}^{\star}\left(C l^{\star}\left(B_{2}\right)\right)\right)\right)\right] \\
& \subseteq F^{-}\left(C l^{\star}\left(B_{1}\right)\right) \cup F^{+}\left(C l^{\star}\left(B_{2}\right)\right)
\end{aligned}
$$

for every subsets $B_{1}, B_{2}$ of $Y$;

(5)

$$
\begin{aligned}
& F^{+}\left(\operatorname{Int}^{\star}\left(B_{1}\right)\right) \cap F^{-}\left(\operatorname{Int}^{\star}\left(B_{2}\right)\right) \\
& \subseteq \operatorname{Int}^{\star}\left[F^{+}\left(\operatorname{Int}\left(\operatorname{Cl}^{\star}\left(\operatorname{Int}^{\star}\left(B_{1}\right)\right)\right)\right)\right. \\
& \cap F^{-}\left(\operatorname{Int}\left(\operatorname{Cl}^{\star}\left(\operatorname{Int}^{\star}\left(B_{2}\right)\right)\right)\right]
\end{aligned}
$$

for every subsets $B_{1}, B_{2}$ of $Y$.

Proof. (1) $\Rightarrow(2)$ : Let $V_{1}, V_{2}$ be any $\star$-open sets of $Y$ such that $x \in F^{+}\left(V_{1}\right) \cap F^{-}\left(V_{2}\right)$. Then

$$
F(x) \in V_{1}^{+} \cap V_{2}^{-}
$$

and hence there exists a $\star$-open set $U$ of $X$ containing $x$ such that $F(U) \subseteq \operatorname{Int}\left(\mathrm{Cl}^{\star}\left(V_{1}\right)\right)$ and

$$
F(z) \cap \operatorname{Int}\left(\mathrm{Cl}^{\star}\left(V_{2}\right)\right) \neq \emptyset
$$

for each $z \in U$. Thus,

$$
U \subseteq F^{+}\left(\operatorname{Int}\left(\mathrm{Cl}^{\star}\left(V_{1}\right)\right)\right) \cap F^{-}\left(\operatorname{Int}\left(\mathrm{Cl}^{\star}\left(V_{2}\right)\right)\right)
$$

and hence

$$
x \in \operatorname{Int}^{\star}\left[F^{+}\left(\operatorname{Int}\left(\mathrm{Cl}^{\star}\left(V_{1}\right)\right)\right) \cap F^{-}\left(\operatorname{Int}\left(\mathrm{Cl}^{\star}\left(V_{2}\right)\right)\right)\right] .
$$

This shows that

$$
\begin{aligned}
& F^{+}\left(V_{1}\right) \cap F^{-}\left(V_{2}\right) \\
& \subseteq \operatorname{Int}^{\star}\left[F^{+}\left(\operatorname{Int}\left(\mathrm{Cl}^{\star}\left(V_{1}\right)\right)\right) \cap F^{-}\left(\operatorname{Int}\left(\mathrm{Cl}^{\star}\left(V_{2}\right)\right)\right)\right] .
\end{aligned}
$$

(2) $\Rightarrow(3)$ : Let $K_{1}, K_{2}$ be any $\star$-closed sets of $Y$. Then $Y-K_{1}$ and $Y-K_{2}$ are $\star$-open sets of $Y$ and by (2), we have

$$
\begin{aligned}
X- & {\left[F^{-}\left(K_{1}\right) \cup F^{+}\left(K_{2}\right)\right] } \\
= & {\left[X-F^{-}\left(K_{1}\right)\right] \cap\left[X-F^{+}\left(K_{2}\right)\right] } \\
= & F^{+}\left(Y-K_{1}\right) \cap F^{-}\left(Y-K_{2}\right) \\
\subseteq & \operatorname{Int}^{\star}\left[F^{+}\left(\operatorname{Int}\left(\mathrm{Cl}^{\star}\left(Y-K_{1}\right)\right)\right)\right. \\
& \left.\cap F^{-}\left(\operatorname{Int}\left(\mathrm{Cl}^{\star}\left(Y-K_{2}\right)\right)\right)\right] \\
= & \operatorname{Int}^{\star}\left[\left(X-F^{-}\left(\mathrm{Cl}\left(\operatorname{Int}^{\star}\left(K_{1}\right)\right)\right)\right)\right. \\
& \left.\cap\left(X-F^{+}\left(\operatorname{Cl}\left(\operatorname{Int}{ }^{\star}\left(K_{2}\right)\right)\right)\right)\right] \\
= & \operatorname{Int}^{\star}\left[X-\left[F^{-}\left(\mathrm{Cl}\left(\operatorname{Int}^{\star}\left(K_{1}\right)\right)\right)\right.\right. \\
& \left.\left.\cup F^{+}\left(\operatorname{Cl}\left(\operatorname{Int}^{\star}\left(K_{2}\right)\right)\right)\right]\right] \\
= & X-\operatorname{Cl}{ }^{\star}\left[F^{-}\left(\mathrm{Cl}\left(\operatorname{Int}^{\star}\left(K_{1}\right)\right)\right)\right. \\
& \left.\cup F^{+}\left(\operatorname{Cl}\left(\operatorname{Int}^{\star}\left(K_{2}\right)\right)\right)\right]
\end{aligned}
$$

and hence

$$
\begin{aligned}
& \mathrm{Cl}^{\star}\left[F^{-}\left(\mathrm{Cl}\left(\operatorname{Int}^{\star}\left(K_{1}\right)\right)\right) \cup F^{+}\left(\mathrm{Cl}\left(\operatorname{Int}^{\star}\left(K_{2}\right)\right)\right)\right] \\
& \subseteq F^{-}\left(K_{1}\right) \cup F^{+}\left(K_{2}\right) .
\end{aligned}
$$

$(3) \Rightarrow(4)$ : Let $B_{1}, B_{2}$ be any subsets of $Y$. Then $\mathrm{Cl}^{\star}\left(B_{1}\right)$ and $\mathrm{Cl}^{\star}\left(B_{2}\right)$ are $\star$-closed in $Y$ and by $(3)$, $\mathrm{Cl}^{\star}\left[F^{-}\left(\mathrm{Cl}\left(\operatorname{Int}^{\star}\left(\mathrm{Cl}^{\star}\left(B_{1}\right)\right)\right)\right) \cup F^{+}\left(\mathrm{Cl}\left(\operatorname{Int}^{\star}\left(\mathrm{Cl}^{\star}\left(B_{2}\right)\right)\right)\right)\right]$ $\subseteq F^{-}\left(\mathrm{Cl}^{\star}\left(B_{1}\right)\right) \cup F^{+}\left(\mathrm{Cl}^{\star}\left(B_{2}\right)\right)$.

$(4) \Rightarrow(5)$ : Let $B_{1}, B_{2}$ be any subsets of $Y$. By (4), we have

$$
\begin{aligned}
& F^{-}\left(\operatorname{Int}^{\star}\left(B_{1}\right)\right) \cap F^{+}\left(\operatorname{Int}^{\star}\left(B_{2}\right)\right) \\
& =X-\left[F^{+}\left(\mathrm{Cl}^{\star}\left(Y-B_{1}\right)\right) \cup F^{-}\left(\mathrm{Cl}^{\star}\left(Y-B_{2}\right)\right)\right] \\
& \subseteq X-\mathrm{Cl}^{\star}\left[F^{+}\left(\mathrm{Cl}\left(\operatorname{Int}^{\star}\left(\mathrm{Cl}^{\star}\left(Y-B_{1}\right)\right)\right)\right)\right. \\
& \left.\cup F^{-}\left(\mathrm{Cl}\left(\operatorname{Int}^{\star}\left(\mathrm{Cl}^{\star}\left(Y-B_{2}\right)\right)\right)\right)\right] \\
& =X-\mathrm{Cl}^{\star}\left[F^{+}\left(Y-\operatorname{Int}\left(\mathrm{Cl}^{\star}\left(\operatorname{Int}^{\star}\left(B_{1}\right)\right)\right)\right)\right. \\
& \left.\cup F^{-}\left(Y-\operatorname{Int}\left(\mathrm{Cl}^{\star}\left(\operatorname{Int}^{\star}\left(B_{2}\right)\right)\right)\right)\right] \\
& =X-\mathrm{Cl}^{\star}\left[\left(X-F^{-}\left(\operatorname{Int}\left(\mathrm{Cl}^{\star}\left(\operatorname{Int}^{\star}\left(B_{1}\right)\right)\right)\right)\right)\right. \\
& \left.\cup\left(X-F^{+}\left(\operatorname{Int}\left(\mathrm{Cl}^{\star}\left(\operatorname{Int}^{\star}\left(B_{2}\right)\right)\right)\right)\right)\right] \\
& =X-\mathrm{Cl}^{\star}\left[X-\left[F^{-}\left(\operatorname{Int}\left(\mathrm{Cl}^{\star}\left(\operatorname{Int}^{\star}\left(B_{1}\right)\right)\right)\right)\right.\right. \\
& \left.\left.\cap F^{+}\left(\operatorname{Int}\left(\mathrm{Cl}^{\star}\left(\operatorname{Int}^{\star}\left(B_{2}\right)\right)\right)\right)\right]\right] \\
& =\operatorname{Int}^{\star}\left[F^{-}\left(\operatorname{Int}\left(\mathrm{Cl}^{\star}\left(\operatorname{Int}^{\star}\left(B_{1}\right)\right)\right)\right)\right. \\
& \left.\cap F^{+}\left(\operatorname{Int}\left(\mathrm{Cl}^{\star}\left(\operatorname{Int}^{\star}\left(B_{2}\right)\right)\right)\right)\right] \text {. }
\end{aligned}
$$
such that $x \in F^{+}\left(V_{1}\right) \cap F^{-}\left(V_{2}\right)$. By (2), we have

$$
\begin{aligned}
& x \in F^{+}\left(V_{1}\right) \cap F^{-}\left(V_{2}\right) \\
& \subseteq \operatorname{Int}^{\star}\left[F^{+}\left(\operatorname{Int}\left(\mathrm{Cl}^{\star}\left(V_{1}\right)\right)\right) \cap F^{-}\left(\operatorname{Int}\left(\mathrm{Cl}^{\star}\left(V_{2}\right)\right)\right)\right] .
\end{aligned}
$$


Then, there exists a $\star$-open set $U$ of $X$ such that

$$
x \in U \subseteq F^{+}\left(\operatorname{Int}\left(\mathrm{Cl}^{\star}\left(V_{1}\right)\right)\right) \cap F^{-}\left(\operatorname{Int}\left(\mathrm{Cl}^{\star}\left(V_{2}\right)\right)\right) .
$$

Thus, $F(U) \subseteq \operatorname{Int}\left(\mathrm{Cl}^{\star}\left(V_{1}\right)\right)$ and

$$
F(z) \cap \operatorname{Int}\left(\mathrm{Cl}^{\star}\left(V_{2}\right)\right) \neq \emptyset
$$

for every $z \in U$. This shows that $F$ is almost $\imath^{\star}$ continuous.

\section{On characterizations of weakly $\imath^{\star}$ - continuous multifunctions}

In this section, we introduce the concept of weakly $\imath^{\star}$-continuous multifunctions. Moreover, some characterizations of weakly $\imath^{\star}$-continuous multifunctions are discussed.

Definition 9. A multifunction $F:(X, \tau, \mathscr{I}) \rightarrow$ $(Y, \sigma, \mathscr{J})$ is said to be weakly $\imath^{\star}$-continuous if for each $x \in X$ and each $\star$-open set $V_{1}, V_{2}$ of $Y$ such that $F(x) \in V_{1}^{+} \cap V_{2}^{-}$, there exists a -open set $U$ of $X$ containing $x$ such that $F(U) \subseteq C l^{\star}\left(V_{1}\right)$ and $F(z) \cap C l^{\star}\left(V_{2}\right) \neq \emptyset$ for every $z \in U$.

Remark 10. For a multifunction $F:(X, \tau, \mathscr{I}) \rightarrow$ $(Y, \sigma, \mathscr{J})$, the following implication holds:

$$
\text { almost } \imath^{\star} \text {-continuity } \Rightarrow \text { weak } \imath^{\star} \text {-continuity. }
$$

The converse of the implication is not true in general. We give example for the implication as follows.

Example 11. Let $X=\{1,2,3\}$ with a topology $\tau=$ $\{\emptyset,\{2\},\{1,3\}, X\}$ and an ideal $\mathscr{I}=\{\emptyset\}$. Let $Y=$ $\{a, b, c\}$ with a topology $\sigma=\{\emptyset,\{a\},\{b\},\{a, b\}, Y\}$ and an ideal $\mathscr{J}=\{\emptyset,\{c\}\}$. A multifunction $F:(X, \tau, \mathscr{I}) \rightarrow(Y, \sigma, \mathscr{J})$ is defined as follows: $F(1)=\{a\}, F(2)=\{b\}$ and $F(3)=\{a, c\}$. Then $F$ is weakly $\imath^{\star}$-continuous but $F$ is not almost $\imath^{\star}$ continuous.

Theorem 12. For a multifunction $F:(X, \tau, \mathscr{I}) \rightarrow$ $(Y, \sigma, \mathscr{J})$, the following properties are equivalent:

(1) F is weakly $\imath^{\star}$-continuous;

$$
\begin{aligned}
& F^{+}\left(V_{1}\right) \cap F^{-}\left(V_{2}\right) \\
& \subseteq \operatorname{Int}^{\star}\left[F^{+}\left(C l^{\star}\left(V_{1}\right)\right) \cap F^{-}\left(C l^{\star}\left(V_{2}\right)\right)\right]
\end{aligned}
$$

for every $\star$-open sets $V_{1}, V_{2}$ of $Y$;
(3)

$$
\begin{aligned}
& C l^{\star}\left[F^{-}\left(\operatorname{Int} t^{\star}\left(K_{1}\right)\right) \cup F^{+}\left(\operatorname{Int} t^{\star}\left(K_{2}\right)\right)\right] \\
& \subseteq F^{-}\left(K_{1}\right) \cup F^{+}\left(K_{2}\right)
\end{aligned}
$$

for every $\star$-closed sets $K_{1}, K_{2}$ of $Y$;

(4)

$$
\begin{aligned}
& C l^{\star}\left[F^{-}\left(\operatorname{Int}^{\star}\left(C l^{\star}\left(B_{1}\right)\right)\right) \cup F^{+}\left(\operatorname{Int}^{\star}\left(C l^{\star}\left(B_{2}\right)\right)\right)\right] \\
& \subseteq F^{-}\left(C l^{\star}\left(B_{1}\right)\right) \cup F^{+}\left(C l^{\star}\left(B_{2}\right)\right)
\end{aligned}
$$

for every subsets $B_{1}, B_{2}$ of $Y$;

(5)

$$
\begin{aligned}
& F^{+}\left(\operatorname{Int}^{\star}\left(B_{1}\right)\right) \cap F^{-}\left(\operatorname{Int}^{\star}\left(B_{2}\right)\right) \\
& \subseteq \operatorname{Int}^{\star}\left[F^{+}\left(C l^{\star}\left(B_{1}\right)\right) \cap F^{-}\left(C l^{\star}\left(B_{2}\right)\right)\right]
\end{aligned}
$$

for every subsets $B_{1}, B_{2}$ of $Y$;

(6)

$$
\begin{aligned}
& C l^{\star}\left[F^{-}\left(V_{1}\right) \cup F^{+}\left(V_{2}\right)\right] \\
& \subseteq F^{-}\left(C l^{\star}\left(V_{1}\right)\right) \cup F^{+}\left(C l^{\star}\left(V_{2}\right)\right)
\end{aligned}
$$

for every $\star$-open sets $V_{1}, V_{2}$ of $Y$.

Proof. (1) $\Rightarrow(2)$ : Let $V_{1}, V_{2}$ be any $\star$-open sets of $Y$ such that $x \in F^{+}\left(V_{1}\right) \cap F^{-}\left(V_{2}\right)$. Then

$$
F(x) \in V_{1}^{+} \cap V_{2}^{+}
$$

and hence there exists a $\star$-open set $U$ of $X$ containing $x$ such that $F(U) \subseteq \operatorname{Int}\left(\mathrm{Cl}^{\star}\left(V_{1}\right)\right)$ and

$$
F(z) \cap \operatorname{Int}\left(\mathrm{Cl}^{\star}\left(V_{2}\right)\right) \neq \emptyset
$$

for each $z \in U$. Thus,

$$
x \in U \subseteq F^{+}\left(\mathrm{Cl}^{\star}\left(V_{1}\right)\right) \cap F^{-}\left(\mathrm{Cl}^{\star}\left(V_{2}\right)\right)
$$

and hence $\left.x \in \operatorname{Int}^{\star}\left[F^{+}\left(\mathrm{Cl}^{\star}\left(V_{1}\right)\right) \cap F^{-}\left(\mathrm{Cl}^{\star}\left(V_{2}\right)\right)\right)\right]$. Consequently, we obtain

$$
\begin{aligned}
& F^{+}\left(V_{1}\right) \cap F^{-}\left(V_{2}\right) \\
& \left.\subseteq \operatorname{Int}^{\star}\left[F^{+}\left(\mathrm{Cl}^{\star}\left(V_{1}\right)\right) \cap F^{-}\left(\mathrm{Cl}^{\star}\left(V_{2}\right)\right)\right)\right] .
\end{aligned}
$$

$(2) \Rightarrow(3)$ : Let $K_{1}, K_{2}$ be any $\star$-closed sets of $Y$. Then $Y-K_{1}$ and $Y-K_{2}$ are $\star$-open sets in $Y$. By (2), we have

$$
\begin{aligned}
& X-\left(F^{-}\left(K_{1}\right) \cup F^{+}\left(K_{2}\right)\right) \\
& =\left(X-F^{-}\left(K_{1}\right)\right) \cap\left(X-F^{+}\left(K_{2}\right)\right) \\
& =F^{+}\left(Y-K_{1}\right) \cap F^{-}\left(Y-K_{2}\right) \\
& \subseteq \operatorname{Int}^{\star}\left[F^{+}\left(\mathrm{Cl}^{\star}\left(Y-K_{1}\right)\right) \cap F^{-}\left(\mathrm{Cl}^{\star}\left(Y-K_{2}\right)\right)\right] \\
& =\operatorname{Int}^{\star}\left[\left(X-F^{-}\left(\operatorname{Int}^{\star}\left(K_{1}\right)\right)\right) \cap\left(X-F^{+}\left(\operatorname{Int}^{\star}\left(K_{2}\right)\right)\right)\right] \\
& =\operatorname{Int}^{\star}\left[X-\left[F^{-}\left(\operatorname{Int}^{\star}\left(K_{1}\right)\right) \cup F^{+}\left(\operatorname{Int}^{\star}\left(K_{2}\right)\right)\right]\right] \\
& =X-\mathrm{Cl}^{\star}\left[F^{-}\left(\operatorname{Int}^{\star}\left(K_{1}\right)\right) \cup F^{+}\left(\operatorname{Int}^{\star}\left(K_{2}\right)\right)\right]
\end{aligned}
$$


and hence

$$
\begin{aligned}
& \mathrm{Cl}^{\star}\left[F^{-}\left(\operatorname{Int}^{\star}\left(K_{1}\right)\right) \cup F^{+}\left(\operatorname{Int}^{\star}\left(K_{2}\right)\right)\right] \\
& \subseteq F^{-}\left(K_{1}\right) \cup F^{+}\left(K_{2}\right) .
\end{aligned}
$$

$(3) \Rightarrow(4)$ : Let $B_{1}, B_{2}$ be any subsets of $Y$. Then $\mathrm{Cl}^{\star}\left(B_{1}\right)$ and $\mathrm{Cl}^{\star}\left(B_{2}\right)$ are $\star$-closed in $Y$ and by (3),

$$
\begin{aligned}
& \mathrm{Cl}^{\star}\left[F^{-}\left(\operatorname{Int}^{\star}\left(\mathrm{Cl}^{\star}\left(B_{1}\right)\right)\right) \cup F^{+}\left(\operatorname{Int}^{\star}\left(\mathrm{Cl}^{\star}\left(B_{2}\right)\right)\right)\right] \\
& \subseteq F^{-}\left(\mathrm{Cl}^{\star}\left(B_{1}\right)\right) \cup F^{+}\left(\mathrm{Cl}^{\star}\left(B_{2}\right)\right) .
\end{aligned}
$$

$(4) \Rightarrow(5)$ : Let $B_{1}, B_{2}$ be any subsets of $Y$. By (4), we have

$$
\begin{aligned}
F^{-} & \left(\operatorname{Int}^{\star}\left(B_{1}\right)\right) \cap F^{+}\left(\operatorname{Int}^{\star}\left(B_{2}\right)\right) \\
= & X-\left[F^{+}\left(\mathrm{Cl}^{\star}\left(Y-B_{1}\right)\right) \cup F^{-}\left(\mathrm{Cl}^{\star}\left(Y-B_{2}\right)\right)\right] \\
\subseteq & X-\mathrm{Cl}^{\star}\left[F^{+}\left(\operatorname{Int}^{\star}\left(\mathrm{Cl}^{\star}\left(Y-B_{1}\right)\right)\right)\right. \\
& \left.\cup F^{-}\left(\operatorname{Int}^{\star}\left(\mathrm{Cl}^{\star}\left(Y-B_{2}\right)\right)\right)\right] \\
= & X-\mathrm{Cl}^{\star}\left[F^{+}\left(Y-\mathrm{Cl}^{\star}\left(\operatorname{Int}^{\star}\left(B_{1}\right)\right)\right)\right. \\
& \left.\cup F^{-}\left(Y-\mathrm{Cl}^{\star}\left(\operatorname{Int}^{\star}\left(B_{2}\right)\right)\right)\right] \\
= & X-\mathrm{Cl}^{\star}\left[\left(X-F^{-}\left(\mathrm{Cl}^{\star}\left(\operatorname{Int}^{\star}\left(B_{1}\right)\right)\right)\right)\right. \\
& \left.\cup\left(X-F^{+}\left(\mathrm{Cl}^{\star}\left(\operatorname{Int}^{\star}\left(B_{2}\right)\right)\right)\right)\right] \\
= & X-\mathrm{Cl}^{\star}\left[X-\left[F^{-}\left(\operatorname{Int}^{\star}\left(\mathrm{Cl}^{\star}\left(B_{1}\right)\right)\right)\right.\right. \\
& \left.\left.\cap F^{+}\left(\mathrm{Cl}^{\star}\left(\operatorname{Int}^{\star}\left(B_{2}\right)\right)\right)\right]\right] \\
= & \operatorname{Int}^{\star}\left[F^{-}\left(\mathrm{Cl}^{\star}\left(\operatorname{Int}^{\star}\left(B_{1}\right)\right)\right) \cap F^{+}\left(\mathrm{Cl}^{\star}\left(\operatorname{Int}^{\star}\left(B_{2}\right)\right)\right)\right] .
\end{aligned}
$$

Thus,

$$
\begin{aligned}
& F^{+}\left(\operatorname{Int}^{\star}\left(B_{1}\right)\right) \cap F^{-}\left(\operatorname{Int}^{\star}\left(B_{2}\right)\right) \\
& \subseteq \operatorname{Int}^{\star}\left[F^{+}\left(\operatorname{Cl}^{\star}\left(B_{1}\right)\right) \cap F^{-}\left(\mathrm{Cl}^{\star}\left(B_{2}\right)\right)\right] .
\end{aligned}
$$

(5) $\Rightarrow(2)$ : This is obvious.

$(2) \Rightarrow(1)$ : Let $V_{1}, V_{2}$ be any $\star$-open sets of $Y$ such that $x \in F^{+}\left(V_{1}\right) \cap F^{-}\left(V_{2}\right)$. By $(2)$, we have

$$
\begin{aligned}
& x \in F^{+}\left(V_{1}\right) \cap F^{-}\left(V_{2}\right) \\
& \left.\subseteq \operatorname{Int}^{\star}\left[F^{+}\left(\mathrm{Cl}^{\star}\left(V_{1}\right)\right) \cap F^{-}\left(\mathrm{Cl}^{\star}\left(V_{2}\right)\right)\right)\right] .
\end{aligned}
$$

Then, there exists a $\star$-open set $U$ of $X$ such that $x \in U \subseteq F^{+}\left(\mathrm{Cl}^{\star}\left(V_{1}\right)\right) \cap F^{-}\left(\mathrm{Cl}^{\star}\left(V_{2}\right)\right)$. Therefore, $F(U) \subseteq \mathrm{Cl}^{\star}\left(V_{1}\right)$ and $F(z) \cap \mathrm{Cl}^{\star}\left(V_{2}\right) \neq \emptyset$ for every $z \in U$. This shows that $F$ is weakly $\imath^{\star}$-continuous.

$(4) \Rightarrow(6)$ : Let $V_{1}, V_{2}$ be any $\star$-open sets of $Y$. By (4), we have

$$
\begin{aligned}
& \mathrm{Cl}^{\star}\left[F^{-}\left(V_{1}\right) \cup F^{+}\left(V_{2}\right)\right] \\
& \subseteq \mathrm{Cl}^{\star}\left[F^{-}\left(\operatorname{Int}^{\star}\left(\mathrm{Cl}^{\star}\left(V_{1}\right)\right)\right) \cup F^{+}\left(\operatorname{Int}^{\star}\left(\mathrm{Cl}^{\star}\left(V_{2}\right)\right)\right)\right] \\
& \subseteq F^{-}\left(\mathrm{Cl}^{\star}\left(V_{1}\right)\right) \cup F^{+}\left(\mathrm{Cl}^{\star}\left(V_{2}\right)\right) .
\end{aligned}
$$

$(6) \Rightarrow(2)$ : Let $V_{1}, V_{2}$ be any $\star$-open sets of $Y$. By (6), we have

$$
\begin{aligned}
F^{+}\left(V_{1}\right) \cap F^{-}\left(V_{2}\right) \\
\subseteq \\
=F^{+}\left(\operatorname{Int}^{\star}\left(\mathrm{Cl}^{\star}\left(V_{1}\right)\right)\right) \cap F^{-}\left(\operatorname{Int}^{\star}\left(\mathrm{Cl}^{\star}\left(V_{2}\right)\right)\right) \\
=X-\left[F^{-}\left(\mathrm{Cl}^{\star}\left(Y-\mathrm{Cl}^{\star}\left(V_{1}\right)\right)\right)\right. \\
\left.\quad \cup F^{+}\left(\mathrm{Cl}^{\star}\left(Y-\mathrm{Cl}^{\star}\left(V_{2}\right)\right)\right)\right] \\
\subseteq X-\mathrm{Cl}^{\star}\left[F^{-}\left(Y-\mathrm{Cl}^{\star}\left(V_{1}\right)\right) \cup F^{+}\left(Y-\mathrm{Cl}^{\star}\left(V_{2}\right)\right)\right] \\
=\operatorname{Int}^{\star}\left[F^{+}\left(\mathrm{Cl}^{\star}\left(V_{1}\right)\right) \cap F^{-}\left(\mathrm{Cl}^{\star}\left(V_{2}\right)\right)\right] .
\end{aligned}
$$

Definition 13. [4] A point $x$ in an ideal topological space $(X, \tau, \mathscr{I})$ is called $a \star_{\theta}$-cluster point of $A$ if $\left.C l^{\star}(U)\right) \cap A \neq \emptyset$ for every $\star$-open set $U$ of $X$ containing $x$. The set of all $\star_{\theta}$-cluster points of $A$ is called the $\star_{\theta}$-closure of $A$ and is denoted by $\star_{\theta} C l(A)$.

Definition 14. [4] A subset $A$ of an ideal topological space $(X, \tau, \mathscr{I})$ is called

(1) $\star_{\theta}$-closed if $\star_{\theta} \operatorname{Cl}(A)=A$.

(2) $\star_{\theta}$-open if its complement is $\star_{\theta}$-closed.

Lemma 15. [4] For a subset $A$ of an ideal topological space $(X, \tau, \mathscr{I})$, the following properties hold:

(1) If $A$ is $\star$-open in $X$, then $C l^{\star}(A)={ }_{{ }_{\theta}} C l(A)$.

(2) $\star_{\theta} \operatorname{Cl}(A)$ is $\star$-closed in $X$.

Theorem 16. For a multifunction $F:(X, \tau, \mathscr{I}) \rightarrow$ $(Y, \sigma, \mathscr{J})$, the following properties are equivalent:

(1) F is weakly $\imath^{\star}$-continuous;

(2)

$$
\begin{aligned}
& C l^{\star}\left[F^{-}\left(\operatorname{Int} t^{\star}\left(\star_{\theta} C l\left(B_{1}\right)\right)\right) \cap F^{+}\left(\operatorname{Int} t^{\star}\left(\star_{\theta} C l\left(B_{2}\right)\right)\right)\right] \\
& \subseteq F^{-}\left(\star_{\theta} C l\left(B_{1}\right)\right) \cup F^{+}\left(\star_{\theta} C l\left(B_{2}\right)\right)
\end{aligned}
$$

for every subsets $B_{1}, B_{2}$ of $Y$;

(3)

$$
\begin{aligned}
& C l^{\star}\left[F^{-}\left(\operatorname{Int}^{\star}\left(\operatorname{Cl}^{\star}\left(B_{1}\right)\right)\right) \cup F^{+}\left(\operatorname{Int}^{\star}\left(\mathrm{Cl}^{\star}\left(B_{2}\right)\right)\right)\right] \\
& \subseteq F^{-}\left(\star_{\theta} C l\left(B_{1}\right)\right) \cup F^{+}\left(\star_{\theta} C l\left(B_{2}\right)\right)
\end{aligned}
$$

for every subsets $B_{1}, B_{2}$ of $Y$;

(4)

$$
\begin{aligned}
& C l^{\star}\left[F^{-}\left(\operatorname{Int^{\star }}\left(C l^{\star}\left(V_{1}\right)\right)\right) \cup F^{+}\left(\operatorname{Int} t^{\star}\left(C l^{\star}\left(V_{2}\right)\right)\right)\right] \\
& \subseteq F^{-}\left(C l^{\star}\left(V_{1}\right)\right) \cup F^{+}\left(C l^{\star}\left(V_{2}\right)\right)
\end{aligned}
$$

for every $\star$-open sets $V_{1}, V_{2}$ of $Y$; 
(5)

$$
\begin{aligned}
& C l^{\star}\left[F^{-}\left(\operatorname{Int^{\star }}\left(C l^{\star}\left(V_{1}\right)\right)\right) \cup F^{+}\left(\operatorname{Int}^{\star}\left(C l^{\star}\left(V_{2}\right)\right)\right)\right] \\
& \subseteq F^{-}\left(C l^{\star}\left(V_{1}\right)\right) \cup F^{+}\left(C l^{\star}\left(V_{2}\right)\right)
\end{aligned}
$$

for every $\mathscr{J}^{\star}$-preopen sets $V_{1}, V_{2}$ of $Y$;

(6)

$$
\begin{aligned}
& C l^{\star}\left[F^{-}\left(\operatorname{Int} t^{\star}\left(K_{1}\right)\right) \cup F^{+}\left(\operatorname{Int}^{\star}\left(K_{2}\right)\right)\right] \\
& \subseteq F^{-}\left(K_{1}\right) \cup F^{+}\left(K_{2}\right)
\end{aligned}
$$

for every $R$ - $\mathscr{J}^{\star}$-closed sets $K_{1}, K_{2}$ of $Y$.

Proof. (1) $\Rightarrow(2)$ : Let $B_{1}, B_{2}$ be any subset of $Y$. Then $\star_{\theta} \mathrm{Cl}\left(B_{1}\right)$ and $\star_{\theta} \mathrm{Cl}\left(B_{2}\right)$ are $\star$-closed in $Y$. By Theorem 12, we have

$$
\begin{aligned}
& \mathrm{Cl}^{\star}\left[F^{-}\left(\operatorname{Int}^{\star}\left(\star_{\theta} \mathrm{Cl}\left(B_{1}\right)\right)\right) \cup F^{+}\left(\operatorname{Int}^{\star}\left(\star_{\theta} \mathrm{Cl}\left(B_{2}\right)\right)\right)\right] \\
& \subseteq F^{-}\left(\star_{\theta} \mathrm{Cl}\left(B_{1}\right)\right) \cup F^{+}\left(\star_{\theta} \mathrm{Cl}\left(B_{2}\right)\right) .
\end{aligned}
$$

$(2) \Rightarrow(3)$ : This is obvious since $\mathrm{Cl}^{\star}(B) \subseteq$ $\star_{\theta} \mathrm{Cl}(B)$ for every subset $B$ of $Y$.

$(3) \Rightarrow(4)$ : This is obvious since $\mathrm{Cl}^{\star}(V)=$ $\star_{\theta} \mathrm{Cl}(V)$ for every $\star$-open set $V$ of $Y$.

$(4) \Rightarrow(5)$ : Let $V_{1}, V_{2}$ be any $\mathscr{J}^{\star}$-preopen sets of $Y$. Since $V_{i} \subseteq \operatorname{Int}^{\star}\left(\mathrm{Cl}^{\star}\left(V_{i}\right)\right)$, we have

$$
\mathrm{Cl}^{\star}\left(V_{i}\right)=\mathrm{Cl}^{\star}\left(\operatorname{Int}^{\star}\left(\mathrm{Cl}^{\star}\left(V_{i}\right)\right)\right)
$$

for $i=1,2$. Now, put $U_{i}=\operatorname{Int}^{\star}\left(\mathrm{Cl}^{\star}\left(V_{i}\right)\right)$, then $U_{i}$ is $\star$-open in $Y$ and $\mathrm{Cl}^{\star}\left(U_{i}\right)=\mathrm{Cl}^{\star}\left(V_{i}\right)$. Therefore, by (4), we have

$$
\begin{aligned}
& \mathrm{Cl}^{\star}\left[F^{-}\left(\operatorname{Int}^{\star}\left(\mathrm{Cl}^{\star}\left(V_{1}\right)\right)\right) \cup F^{+}\left(\operatorname{Int}^{\star}\left(\mathrm{Cl}^{\star}\left(V_{2}\right)\right)\right)\right] \\
& \subseteq F^{-}\left(\mathrm{Cl}^{\star}\left(V_{1}\right)\right) \cup \mathrm{Cl}^{\star}\left(V_{2}\right) .
\end{aligned}
$$

$(5) \Rightarrow(6)$ : Let $K_{1}, K_{2}$ be any $R-\mathscr{J}^{\star}$-closed sets of $Y$. Then $\operatorname{Int}^{\star}\left(K_{1}\right)$ and $\operatorname{Int}^{\star}\left(K_{2}\right)$ are $\mathscr{J}^{\star}$-preopen in $Y$ and by (5),

$$
\begin{aligned}
\mathrm{Cl}^{\star}\left(F^{-}\left[\operatorname{Int}^{\star}\left(K_{1}\right)\right) \cup F^{+}\left(\operatorname{Int}^{\star}\left(K_{2}\right)\right)\right] \\
=\mathrm{Cl}^{\star}\left[F^{-}\left(\operatorname{Int}^{\star}\left(\mathrm{Cl}^{\star}\left(\operatorname{Int}^{\star}\left(K_{1}\right)\right)\right)\right)\right. \\
\left.\quad \cup F^{+}\left(\operatorname{Int}^{\star}\left(\operatorname{Cl}^{\star}\left(\operatorname{Int}^{\star}\left(K_{2}\right)\right)\right)\right)\right] \\
\subseteq F^{-}\left(K_{1}\right) \cup F^{+}\left(K_{2}\right) .
\end{aligned}
$$

$(6) \Rightarrow(1)$ : Let $V_{1}, V_{2}$ be any $\star$-open sets of $Y$. Then $\mathrm{Cl}^{\star}\left(V_{1}\right)$ and $\mathrm{Cl}^{\star}\left(V_{1}\right)$ are $R-\mathscr{J}^{\star}$-closed in $Y$ and by $(6)$, we have

$$
\begin{aligned}
& \mathrm{Cl}^{\star}\left[F^{-}\left(V_{1}\right) \cup F^{+}\left(V_{2}\right)\right] \\
& \subseteq \mathrm{Cl}^{\star}\left[F^{-}\left(\operatorname{Int}^{\star}\left(\mathrm{Cl}^{\star}\left(V_{1}\right)\right)\right) \cup F^{+}\left(\operatorname{Int}^{\star}\left(\mathrm{Cl}^{\star}\left(V_{2}\right)\right)\right)\right] \\
& \subseteq F^{-}\left(\mathrm{Cl}^{\star}\left(V_{1}\right) \cup F^{+}\left(\mathrm{Cl}^{\star}\left(V_{2}\right)\right) .\right.
\end{aligned}
$$

It follows from Theorem 12 that $F$ is weakly $\imath^{\star}$ continuous.
Theorem 17. For a multifunction $F:(X, \tau, \mathscr{I}) \rightarrow$ $(Y, \sigma, \mathscr{J})$, the following properties are equivalent:

(1) F is weakly $\imath^{\star}$-continuous;

$$
\begin{aligned}
& C l^{\star}\left[F^{-}\left(I n t^{\star}\left(C l^{\star}\left(V_{1}\right)\right)\right) \cup F^{+}\left(\operatorname{Int} t^{\star}\left(C l^{\star}\left(V_{2}\right)\right)\right)\right] \\
& \subseteq F^{-}\left(C l^{\star}\left(V_{1}\right)\right) \cup F^{+}\left(C l^{\star}\left(V_{2}\right)\right)
\end{aligned}
$$

for every semi- $\mathscr{J}^{\star}$-preopen sets $V_{1}, V_{2}$ of $Y$;

(3)

$$
\begin{aligned}
& C l^{\star}\left[F^{-}\left(I n t^{\star}\left(C l^{\star}\left(V_{1}\right)\right)\right) \cup F^{+}\left(\operatorname{Int} t^{\star}\left(C l^{\star}\left(V_{2}\right)\right)\right)\right] \\
& \subseteq F^{-}\left(C l^{\star}\left(V_{1}\right)\right) \cup F^{+}\left(C l^{\star}\left(V_{2}\right)\right)
\end{aligned}
$$

for every semi- $\mathscr{J}^{\star}$-open sets $V_{1}, V_{2}$ of $Y$.

Proof. (1) $\Rightarrow(2)$ : Let $V_{1}, V_{2}$ be any semi- $\mathscr{J}^{\star}$ preopen sets of $Y$. Then, we have

$$
V_{i} \subseteq \mathrm{Cl}^{\star}\left(\operatorname{Int}^{\star}\left(\mathrm{Cl}^{\star}\left(V_{i}\right)\right)\right)
$$

and $\mathrm{Cl}^{\star}\left(V_{i}\right)=\mathrm{Cl}^{\star}\left(\operatorname{Int}^{\star}\left(\mathrm{Cl}^{\star}\left(V_{i}\right)\right)\right)$ for $i=1,2$. Since $\mathrm{Cl}^{\star}\left(V_{1}\right)$ and $\mathrm{Cl}^{\star}\left(V_{2}\right)$ are $R-\mathscr{J}^{\star}$-closed in $Y$ and by Theorem 16,

$$
\begin{aligned}
& \mathrm{Cl}^{\star}\left[F^{-}\left(\operatorname{Int}^{\star}\left(\mathrm{Cl}^{\star}\left(V_{1}\right)\right)\right) \cup F^{+}\left(\operatorname{Int}^{\star}\left(\mathrm{Cl}^{\star}\left(V_{2}\right)\right)\right)\right] \\
& \subseteq F^{-}\left(\mathrm{Cl}^{\star}\left(V_{1}\right)\right) \cup F^{+}\left(\mathrm{Cl}^{\star}\left(V_{2}\right)\right) .
\end{aligned}
$$

$(2) \Rightarrow(3)$ : This is obvious since every semi- $\mathscr{J}^{\star}$ open set is semi- $\mathscr{J}^{\star}$-preopen.

$(3) \Rightarrow(1)$ : Let $V_{1}, V_{2}$ be any semi- $\mathscr{J}^{\star}$-preopen sets of $Y$. Then $\mathrm{Cl}^{\star}\left(V_{1}\right)$ and $\mathrm{Cl}^{\star}\left(V_{2}\right)$ are $R-\mathscr{J}^{\star}$ closed sets of $Y$ and hence $\mathrm{Cl}^{\star}\left(V_{2}\right)$ are semi- $\mathscr{J}^{\star}$ open in $Y$. By (3), we have

$$
\begin{aligned}
& \mathrm{Cl}^{\star}\left[F^{-}\left(\operatorname{Int}^{\star}\left(\mathrm{Cl}^{\star}\left(V_{1}\right)\right)\right) \cup F^{+}\left(\operatorname{Int}^{\star}\left(\mathrm{Cl}^{\star}\left(V_{2}\right)\right)\right)\right] \\
& \subseteq F^{-}\left(\mathrm{Cl}^{\star}\left(V_{1}\right)\right) \cup F^{+}\left(\mathrm{Cl}^{\star}\left(V_{2}\right)\right)
\end{aligned}
$$

and by Theorem $16, F$ is weakly $\imath^{\star}$-continuous.

\section{Conclusion}

Topology plays an important role in computational topology for geometric design, computer-aided geometric design, engineering design, information systems, quantum physics, high energy physics and mathematical sciences. The notions of openness and continuity are fundamental concepts for the study and investigation in topological spaces. Besides, the study of continuity has been found to be useful in computer science and digital topology. Several investigations related to generalized open sets have been presented and various forms of continuity types have been 
introduced. Recently, continuity of multifunctions in topological spaces has been researched by many mathematicians. This article deals with the concept of $\imath^{\star}$-continuous multifunctions in ideal topological spaces. Some characterizations of $\imath^{\star}$-continuous multifunctions are obtained. Moreover, the relationships between $\imath^{\star}$-continuity and the other types of continuity for multifunctions are explored. The ideas and results of this article may motivate further research.

Acknowledgements: This research project was financially supported by Mahasarakham University (Fast Track 2021).

\section{References:}

[1] M.-E. Abd El-Monsef, E.-F. Lashien and A.A. Nasef, On $\mathscr{I}$-open sets and $\mathscr{I}$-continuous functions, Kyungpook Math. J. 32, 1992, pp. 2130.

[2] A. Açikgöz, T. Noiri and Ş. Yüksel, A decomposition of continuity in ideal topological spaces, Acta Math. Hungar. 105, 2004, pp. 2737.

[3] C. Berge, Espaces topologiques fonctions multivoques, Dunod, Paris, 1959.

[4] C. Boonpok, Weak quasi continuity for multifunctions in ideal topological spaces, $A d v$. Math., Sci. J. 9(1), 2020, pp. 339-355.

[5] C. Boonpok, On continuous multifunctions in ideal topological spaces, Lobachevskii $J$. Math. 40, 2019, pp. 24-35.

[6] J. Dontchev, Idealization of Granter-Reilly decomposition theorems, arxiv:math. GN/9901017v1, 1999.

[7] G. Freud, Ein Beitrag zu dem Satze von Cantor und Bendixson, Acta Math. Acad. Sci. Hungar. 9, 1958, pp. 333-336.

[8] E. Hatir, A. Keskin and T. Noiri, A note on strong $\beta$ - $\mathscr{I}$-open sets and strongly $\beta-\mathscr{I}$ continuous functions, Acta Math. Hungar. 108, 2005, pp. 87-94.

[9] E. Hatir and T. Noiri, On semi- $\mathscr{I}$-open sets and semi- $\mathscr{I}$-continuous functions, Acta Math. Hungar. 107, 2005, pp. 345-353.

[10] E. Hatir and T. Noiri, On decompositions of continuity via idealization, Acta Math. Hungar. 96, 2002, pp. 341-349.

[11] D. Janković and T.-R. Hamlett, New topologies from old via ideals, Amer. Math. Monthly 97, 1990, pp. 295-310.

[12] K. Kuratowski, Topology, Vol. I, AcademicPress, New York, 1966.
[13] N. Levine, A Decomposition of continuity in topological spaces, Amer. Math. Monthly 68, 1961, pp. 44-46.

[14] T. Noiri and V. Popa, Weakly quasi-continuous multifunctions, Anal. Univ. Timişoara, Ser. Mat. 26, 1988, pp. 33-38.

[15] V. Popa and T. Noiri, Properties of weakly precontinuous multifunctions, Istanbul Univ. Fen. Fa. Mat. Dergisi 57/58, 1998/1999, pp. 41-52.

[16] V. Popa and T. Noiri, On weakly $\beta$-continuous multifunctions, Bui. St. Univ. "Politehnica", Sre. Mat. Fiz. Timişoara 45, 2000, pp. 1-16.

[17] D.-A. Rose, Weak continuity and almost continuity, Internat. J. Math. Math. Sci. 7, 1984, pp. 311-318.

[18] S. Scheinberg, Topologies which generate a complete measure algebra, Advances in Math. 7, 1971, pp. 231-239.

[19] R. Vaidyanathaswamy, The localization theory in set topology, Proc. Indian Acad. Sci. 20, 1944, pp. 51-61.

\section{Creative Commons Attribution License 4.0 (Attribution 4.0 International, CC BY 4.0)}

This article is published under the terms of the Creative Commons Attribution License 4.0 https://creativecommons.org/licenses/by/4.0/deed.en_US 